\title{
Implementation and Performance of the ATLAS Jet Trigger
}

\section{N. Anjos ${ }^{* \dagger}$ on behalf of the ATLAS TDAQ Collaboration}

Laboratório de Instrumentação e Física Experimental de Partículas (LIP), Lisboa, Portugal

E-mail: Nuno.Anjos@cern.ch

This work presents the challenges of the ATLAS jet trigger and describes its implementation and expected performance. The ATLAS trigger is organized in three levels: the first one (L1) is implemented in custom made electronic modules, while the High-Level Trigger (HLT) system, which groups the second (L2) and third (Event Filter, EF) trigger levels, is based on software running in commercial computers. Both L1 and the HLT perform reconstruction of physic objects, like leptons, photons or jets. A precise hadronic calibration is applied at the HLT with the aim of accurately determining the jet energy. This optimizes the behavior of our selection criteria. A dedicated calibration scheme is implemented at L2, while the EF uses offline algorithms. We discuss the strategies used to perform the hadronic calibration for the HLT. We review the current physics signatures, expected rates and efficiencies as obtained from MC studies.

XXth Hadron Collider Physics Symposium

November 16 - 20, 2009

Evian, France

* Speaker.

${ }^{\dagger}$ Work supported by FCT through grant SFRH/BPD/37964/2007. 


\section{Introduction}

ATLAS [1] is a multipurpose experiment at the Large Hadron Collider (LHC). At the LHC nominal luminosity of $10^{34} \mathrm{~cm}^{-2} \mathrm{~s}^{-1}$, with a bunch crossing rate of $40 \mathrm{MHz}$, the resulting volume of data is too large to be stored. The trigger has to select online, with the highest possible efficiency, events considered interesting for physics studies. Only these will be recorded. To meet these requirements, the ATLAS trigger system [2] selects high $p_{T}$ physics objects. In the case of the ATLAS jet trigger [3], a predefined set of $p_{T}$ thresholds and prescales (trigger menu) is used to select the jets. Thus, the main challenge is to accurately measure the jet energy in a short time interval.

The ATLAS trigger is divided in three levels: the first one (L1) is hardware based, while the High-Level Trigger (HLT) system, which groups the second (L2) and third (Event Filter, EF) trigger levels, is software based. The L1 uses custom designed processors that operate with coarse granularity. It applies a sliding window algorithm $(0.8 \times 0.8$ in $(\eta, \phi))$ that identifies local energy maxima in the ATLAS calorimeter. It has a $2 \mu$ s latency and reduces the rate to $75 \mathrm{kHz}$.

The L2 is seeded by the L1, hence it uses as starting point the center of the energy clusters found at L1, reconstructing only a region of the detector, known as Region of Interest (RoI), around the L1 seed. It can operate with full or reduced granularity of the calorimeter, corresponding to energy sums per Front-End-Board (FEB), taking a decision in about $40 \mathrm{~ms}$ and reducing the rate to $2 \mathrm{kHz}$. It runs a simplified cone-like algorithm with radius $\mathrm{R}=0.4$ in $(\eta, \phi)$ to determine the energy weighted center of the cluster, and uses a dedicated fast calibration scheme. The EF uses offline algorithms that may operate seeded by L2 or in full event access. The EF applies the offline calibrations, has a time budget of 4 to $5 \mathrm{~s}$ and reduces the rate to $200 \mathrm{~Hz}$.

\section{Performance of the ATLAS Jet Trigger}

Detailed performance studies of the ATLAS Jet Trigger were done using Monte Carlo simulated data [3]. Here we present only a summary of the main results.

After jet building at L2, the energy scale is set at the EM level, which does not provide an accurate measure of the jet energy. The L2 jet calibration [3] corrects the jet energy scale using longitudinal weighting [3]. For calibration purposes, the energy deposits that constitute the jet are grouped in calorimeter samplings. At L2, two samplings are considered, corresponding to the electromagnetic and hadronic calorimeters. The calibrated jet energy is the weighted sum of the jet energy in each of the samplings, where the weights are considered to have a logarithmic dependency on the energy. The weights are computed for 50 bins of 0.1 in $\eta$, by minimizing the energy difference between the reconstructed and true energies of the L2 jets in simulated di-jet samples. After calibration, the jet energy scale is correct within $2 \%$ for all energies (Fig. 1), and the jet energy resolution is improved by a few \% [3].

The average total processing time for the $\mathrm{L} 2$ jet algorithm was found to be $22 \mathrm{~ms}$, dominated by the data collection, that accounts for $30 \%$ of the time, and the data preparation step (translation of the raw data received from the read out buffers into $\mathrm{C}++$ objects used by the algorithms). Using the reduced $\mathrm{L} 2$ granularity speeds up the whole process by a factor of 2, while keeping a performance similar to the one obtained with full granularity. 

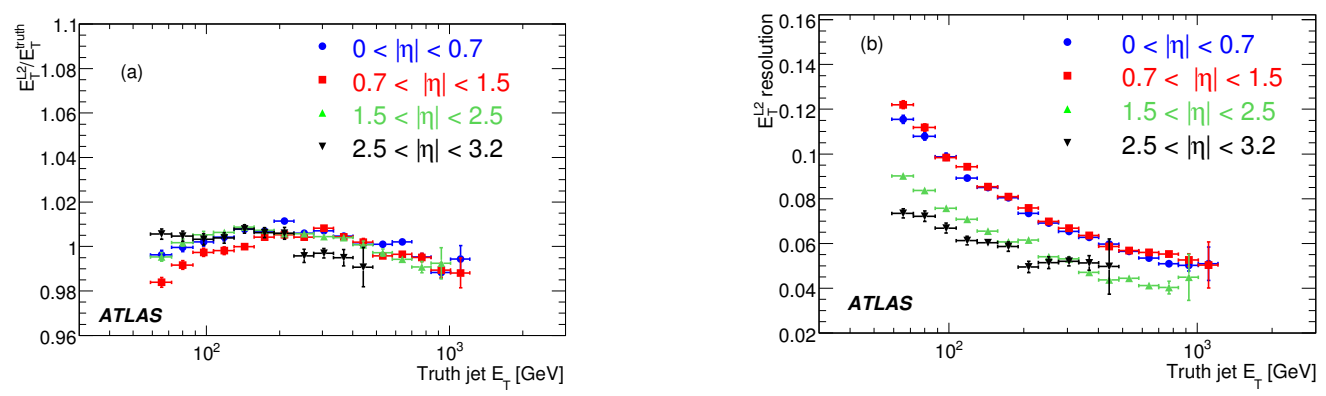

Figure 1: L2 jet energy scale as a function of the truth jet $E_{T}$ (a). L2 jet energy resolution as a function of the truth energy of the jet (b). The results are obtained for four different bins in $\eta$ and after calibration.

The jet trigger menu was optimized to give a flat rate distribution on a wide momentum range, and a single jet rate around $6 \mathrm{~Hz}$. Data driven methods were devised to estimate the efficiency of more complex triggers.

The use of several jet algorithms at EF level was studied. Since in the current setup the reconstruction is limited to the RoI $(1.6 \times 1.6$ in $(\eta, \phi))$, jet energies may differ from the offline. The energy resolutions of EF jets obtained with the ATLAS cone (with radius 0.7 and 0.4), SISCone (with radius 0.5 ) and anti- $k_{T}$ (radius parameter 0.5 ) algorithms were compared with the offline resolution. The cone algorithm with largest radius has the worst resolution. The best resolution was obtained for cone 0.4 and anti- $k_{T}$.

\section{Conclusions}

A 3-level jet trigger was developed in ATLAS. It is able to cover a wide variety of physics topics. The trigger starts with the identification of large transverse energy depositions by the first hardware level, that are later refined at the following two software levels, each employing more refined jet algorithms and calibration schemes. The performance of this trigger system was studied in detail. The processing times of each step were measured and found to be well inside the stringent ATLAS trigger requirements. The jet energy can be reconstructed at the second trigger level with a linearity of $2 \%$ for the whole energy regime covered by the central jet trigger. At the third trigger level, several offline algorithms were compared. The best resolutions are obtained for the cone and anti- $k_{T}$ algorithms with smaller radius.

\section{References}

[1] ATLAS Collaboration, G Aad et al, The ATLAS Experiment at the CERN Large Hadron Collider, JINST 3 S08003 (2008).

[2] ATLAS TDAQ Collaboration, The ATLAS Trigger/DAQ Authorlist, version 3.1, ATL-DAQ-PUB-2009-008 [http: / / cdsweb. cern.ch/record/1207560] .

[3] ATLAS Collaboration, G Aad et al, Expected Performance of the ATLAS Experiment - Detector, Trigger and Physics, CERN-OPEN-2008-020 [arXiv:0901.0512 ]. 